after trephining and the removal of so many fragments of bone. The fall must have been broken in some way, and the patient thus saved.

J. M- aged thirty-one, admitted September 25th, 1863. Family history good, yielding no evidence of any hereditary diathesis. Patient says he has never been laid up by any illness; he is a healthy, robust-looking man. For some years past he has led a very debauched and dissipated life. On the night of the 24th he had been drinking up to a late hour, and, returning home in a state of intoxication, he wandered from the road into an adjacent field, and walked over the edge of a stone quarry, falling a distance of eighty feet. He was discovered some hours afterwards, and brought to the hospital.

On admission, a large lacerated and contused wound was found, slightly above the left frontal eminence. On introducing the finger through this wound, an extensive comminuted fracture was detected in the frontal bone, near the coronal suture, and somewhat to the left of the vertex, encroaching upon the left parietal. The fragments were firmly impacted and considerably depressed. The patient was perfectly conscious, there being no sign whatever of compression of the brain. About six hours after the accident the patient, at his own request, was placed under the influence of chloroform, and Mr. Whipple twice applied the trephine, subsequently removing eighteen fragments of bone, which in the aggregate weighed four drachms. These pieces of bone, when collected and accurately placed in situ, covered a space measuring 3 in. by $1 \frac{7}{3}$ in. in diameter. The longitudinal sinus was opened, from which a rather profuse hæmorrhage ensued. No sutures were used to the wound, a simple water-dressing alone being applied to it, and the shaven scalp kept cool by means of a damp cloth. Ordered to have milk diet; perfect quietude to be enforced; and a purge to be administered in the morning.

Sept. 26th. - Passed a comfortable night; no noticeable headache; face quite natural; tongue somewhat furred; pulse 66 , full.

27th. - Some twitchings of the upper extremity were noticed by the night nurse during sleep. Pupils natural. No sign of paralysis. Appetite moderately good; complains of thirst. Pulse 76. Ordered an effervescing mixture of citric acid and potash.

28th.-Complains of severe pain in the head; tongue much furred; pulse 68. $\mathrm{He}$ is rather more drowsy than usual; pupils somewhat torpid on exposure to a strong light. Bowels freely acted upon by an aperient, affording him great relief.

30th. - Going on favourably. Wound suppurating; discharge rather offensive. To be cleansed with a solution of Condy's fluid, and a poultice to be applied.

Oct. 20th. - No untoward symptom has occurred; the wound is granulating healthily. Appetite good. To have the ordinary diet of the hospital. Allowed to leave his bed.

Dec. 20th. - The wound is now healed, and the patient declares himself "quite well."

\section{STOCKPORT INFIRMARY.}

ENTRANCE OF A PIECE OF LEAD, TWO OUNCES IN WEIGHT, INTO THE CENTRE OF THE BRAIN; DEATH RESULTING TWENTY-SEVEN HOURS AFTER THE RECEIPT OF THE INJURY.

(Under the care of Mr. J. T. PeARsor.)

THe following case is one of much interest. The particulars were forwarded by Mr. J. Peek Richards, the house-surgeon.

A. B - aged fourteen, was admitted into the infirmary on Oct. 11th, 1864, with a compound comminuted fracture of the skull, caused by the passage of a piece of lead through the forehead in the following manner. It appears that the father of the boy had taken a piece of iron piping into the smithy, and in order to seal one of its ends had poured some molten lead down to the bottom. When his back was turned the boy placed the piping in the fire for the purpose of melting the lead, when by some means or other the leaden plug was propelled along it as if from a gun, and struck him on the forehead.

He was brought to the infirmary five hours after the acci. dent, when, on examination, it was found that there was a wound over the right eve communicating with the interior of the skull through an aperture in the frontal bone. I considerable quantity of cerebral matter had exuded from, and two or three small pieces of bone were foumd lying in, the wound. He was semi-conscious, and when spoken to sharply answered. When in bed, if the clothes were drawn off him he quickly replaced them; and any examination of the wound seemed to cause him considerable pain. There was no paralysis, and the breathing never at any time became sterto. rous.

The loose fragments were removed, the head shaved, and a pledget of lint applied to the wound and evaporating lotion to the head. Croton oil was administered, without effect; but after the use of two enemata his bowels were freely opened. He contimued very restless during the night, continually sitting up and attempting to fight some imaginary foe. He once or twice succeeded in remoring the coverings over the wound, so that during the night a considerable quantity of brainmatter escaped. In the morning well-marked symptoms of encephalitis set in; and about six o'clock P.M., or twentyseven hours after the receipt of the injury, he died; his respiration for the last few hours being principally affected.

His friends strenuously objected to his head being opened; but on examining the seat of injury, a cleanly-cut circular aperture (with no staining round about), about the size of a florin, was found in the right side of the frontal bone, about half an inch above the supra-orbital riclge. On passing a probe through the opening to the extent of about four inches in a direction backwards, inwards, and a little downwards, a foreign body conld be felt, which on extraction proved to be the plug of lead in question; it weighed two ounces, and was of a cylindrical shape, a little over an inch long, with a diameter of nearly three-fourths of an inch, both ends being flat like those of a drum.

\section{athotical Sorietics.}

\section{PATHOLOGICAL SOCIETY OF LONDON.}

\section{Dr. Peacock, Presiderst.}

\section{Dr. Dickinson showed two specimens of}

ENTERITIS OCCURRIX WITHOUT KNOWN CAUSE.

The complaint in this form, he said, is very rarely seen in practice. One patient was a boy who was admitted into hospital for retention of urine, and who died with obscure swelling of the belly, constipation, and romiting. On post-mortem examination the whole mucous membrane of the large intestine was found sloughing; there were several perforations in the intestine and pus in the peritoneal cavity. 'The other case, that of a young woman, was similar : the last two feet of the small intestine were sloughing.

Dr. Dickinson also showed a specimen of PERFORATION OF THE STOMACH,

occurring in a young woman who died from the effects of irritant poison eight days after taking it. After death, the contents of the stomach were found in the peritoneal cavity, and a hole as large as a sixpence was found in the stomach; but the absence of any morbid action around the hole, and the relative extent and shape of the rupture of the serous and mucous coats, showed that the rupture had occurred after death. The case was brought forward on account of its importance in a medico-legal point of view.

Dr. WILKs remarked that Dr. Dickinson's first cases were also important in legal medicine, since the occurrence of acute idiopathic enteritis had been denied at the trial of Dr. Smethurst.

Mr. H. LEF mentioned an instance in which he had found the mucous membrane of the rectum sloughing, in a case of secondary syphilis.

The President remarked that the late Dr. Baly, in his lectures before the College of Physicians, had described cases of enteritis similar to those brought forward by Dr. Dickinson.

Dr. QUAIs observed that the stomach displayed by Dr. Dickinson could not have given way by the generation of gas, had it not been previously softened by the irritant poison.

Mr. HoLxes exhibited a

$$
\text { MALFORMATIOS OF THE BLADDER, }
$$

in which there was a congenital evst attached to a bladdur which was otherwise malformed.

Dr. WILKs showed a specimen of LOSS OF THE PCLMOSARY VALVE.

A man, aged thirty-one, came amongst his out-patients suffering from phthisis. He harl at the same time a donble bruit 
heard over the ralves of the heart, and traceable with equal intensity upwards on the lett side. There was no indication in the pulse of aortic ralrular disease, and yet, from the improbabilities of the affection being in the pulmonary artery, it was thought to be due to an aneurismal or other morbid condition of the aorta. He had had an attack of rheumatism ten years before, since which he had been obliged to leare a laborious occupation for that of a cabman. Ifter death, which took place from disorganization of the lungs, the cause of the systolic and diastolic brut was found to be in the pulmonary artery. One only of the sigmoid ralres remained, another had entirely disappeared, and a third had a mere vestige of itself at the seat of attachment. There was no thickening of the healthy valve, or other signs of an inflammatory process erer having been present. The morbid process appeared to be simply an ulcerative one. Two of the aortic valves were slightly adherent, as the result of a former endocarditis. The right ventricle was not enlarged. As regards the origin of the disease some difficulty existed. The fact of the man's health failing after an attack of rheumation ten years before, pointed to that time as the probable period of the occurrence of the mischief; whilst, on the other hand, the absence of all the ordinary signs of endocarditis, with the simple loss of the valves, sugcested a congenital malformation. 'The latter theory would be merely throwing back the time of the occurrence of endocarditis to intra-utenine life, but would be favoured by the fact that affection of the pulmonary valves appears to be more prone to occur at that time than after the child is born.

\section{Mr. H. Surth showed a}

\section{LARGE TLALOTR REMTOVED FROM THE SCIPLLA}

by Mr. Fergusson. Before operation there was much doubt whether the tumour was connected with the bone. Mr. Fergusson was of opinion that it was ; and this turned out to be the case, and the cumour was removed entire with the lower portion of the bone-that below the spine. The operation was difficult on account of a process turning under the neck of the bone, and rumning towards the shoulder-joint. The tumour appeared to be of an inmocent nature.

Or. WInKs brought forward a child, the subject of Constitutional Syphilis, in whom the usual symptoms of the congenital disease were present, and, besides, there was congenital disease of both testes. Dr. Wilks remarked on the rarity of this complication of the hereditary disease. The enlargement of the testicle was in the process of diminution under the inunction of mercury.

Dr. GIBB brought forward a man who had recovered from NECROSIS OF THE CRICOID CARTILAGE.

The patient was forty-nine rears of age, and had expelled portions of the cricoid cartilage that had undergone necrosis. The openings through which these had escaped were observed with the laryngoscope, and a sketeh of them, with the man's history, appeared in the fourteenth rolume of the "Transactions" of the Society. In August last there was much swelling of the false cords, with stridor, congh, and dyspnoa, which necessitated tracheotomy. As was anticipated, the rings were ossified, and the necessary precautions were taken to obviate this. His subsequent progress was most satisfactory, for all the laryngeal symptoms subsided, and the larynx became clear, with cicatricial contraction of those parts through which the necrosed fragments of cartilage had extruded. The voice was good, clear, and loud, as was made manifest to the Society. Dr. Gibb did not think it prudent to dispense with the silver canula at present, owing to the inclemency of the weather. But, in bringing the case before the Society, he stated that it was one of extreme interest, from the fact of the progressive stages of the necrosis, and the manner in which nature took to get rid of the detached portions of cartilage, having been watched with the aid of the laryngoscope.

Dr. GIBE exhibited

PORTIONS OF GSIFIED TRACHEAL RIXGS

expelled some months after tracheotomy, which had been resorted to for chronic gouty disease of the larynx with obstruction. The operation was one of the most complicated and diffout he hal ever witnessed, from the extremely ossified conditiun of the rinss of the trachea, resembling steel almost in its degree of hardness. The patient, an elderly gentleman, is doing rey we!l, and expelled the fragments exhibited through the mouth oi the tube some weeks back.

Mr. HTLkE read a report on two cases of Pigmental or Black lataract shown at a previous meeting by Mr. Windsor. He had fourd the ribres of the lers strophied as usual, and stained with rerchich nigreent.
Mr. Hrstow read a report by Mr. T. Smith, Mr. Toynbee, and himself, on a case of Tumour of the Tympanum. The disease turned out to be of a sebaceous nature, the peculiarity of the case consisting in the development of sebaceous tumours in the interior of the tympanum instead of in the meatus.

Mr. H. Thompsox showed for Dr. Keith a specimen of

CALCLLCS ADHERIYG TO THE COATS OF THE BLADDER.

Mr. Thompson observed that in most of these cases the evidence was derived from the impressions only of surgeons who found certain difficulties in removing a stone. In Dr. Keith's case, the stone adhering to the bladder was removed in the post-mortem examination. The patient was a girl aged sixteen, who died of typhus fever, without symptoms of stone, although some wrinary symptoms had existed. The postmortem was made hastily, and the stone torn away from the walls of the bladder. It was a largish uric-acid stone, and there was a phosphatic patch on one sicle where the stone was adherent. A letter from Dr. Keith was read, in which he explained how this accident might occur, and mentioned that he had found the complication only in two cases out of more than one hundred lithotomies and as many cases of lithotrity.

Mr. H. Thompson showed a

VERT STOUT HAIR-PIN TAKEN FROM THE BLADDER OF A GIRI AGED THIRTEEN,

which he had removed by the high or supra-pubic operation. The patient had come two hundred miles out of the country, and had experienced very little inconvenience from the journey. The shary ends of the pin were embedded through the coats of the vagina, and though it could be seized by the lithotrite it could not be extracted. The high operation was easily per. formed, and the patient has clone well since, three weeks after the operation. Mr. Thompson remarked that the instrument invented by M. Matthieu for removing such hair-pins by traction would not hare acted here on account of the great strength of the pin. This he had ascertained by experiment.

Dr. GREFNHow showed the

ESOPHAGTS, STOMACH, AND ILECAI FROM A CASE OF DIPHTHERIA.

The patient, a boy aged fifteen, died in the Middlesex Hospital during the relapse of the disease, with increase of the throat affection, albuminuria, and romiting. Dr. Greenhow remarked that he had had sereral fatal cases of the same kind in private practice, where he had had no opportunity of malking a post. mortem examination. In the present case there was much injection of the mucous membrane of the stomach and ileum, the solitary glands were enlarged, and pulmonary apoplexy existed in both lungs.

Dr. GREENrow also showed a specimen of SUPRA-RENAL CAPSULAR DISEASE

from a girl aged twelve years and nine months. The patient, after returning from the country, was thought to be embrowned, but showed no symptoms of the disease till a few weeks before her death, when vomiting commenced and soon became constant, and the pulse very rapid and weak. Dr. Greenhow diagnosed from these symptoms the disease called by the name of Addison, though he could not then see much marked dis coloration; but this soon became perceptible, and the patient died with the usual symptoms. On post-mortem examination, a small deposit of unsoftened tubercle was found in each apex; but there was no morbid deposit to account for death in any organ except the supra-renal capsules, which were totally disorganized and adherent to the organs in the neighbourhood. A drawing of the microscopical appearances of the organ, by Dr. Sanderson, was also exhibited. The blood was examined as soon as possible after death, and appeared natural.

Dr. Playfair showed a case of

EXTRA-UTERTYE FETATION IN THE FALLOPIAY TCBE.

The patient died in King's College Hospital from rupture of the tube. The patient was not aware that she was pregnant, and the symptoms (chiefly romiting of blood) were referred to uleer of the stomach-a diagnosis which was thought to be confirmed by the patient's sudden death with collapse. Th $\theta$ foetus seemed to be of about the age of four months.

Dr. JuLres PoLlock showed an instance of

ANEURISY OF THE AORTA PRESSING ON THE PULMONARY ARTERY.

The case during life was obscure. The patient had had winter cough for some years, with palpitation for a few months. There was a loud systolic bruit, which was supposed to be 
mitral. He was rather hoarse, and ha! a little difficulty of swallowing. After some relief from his stay in hospital, he left, and returned after some months with dropsy, increased cough, and dyspnoea. He died with an increase of these symptoms. On post-mortem examination no disease was found in the mitral ralve. The aorta contained sereral aneurismal pouches, one of which pressed so much on the pulmonary artery as almost to close it. This obstruction to the pulmonary circulation accounted for the absence of symptoms of pulmonary congestion during life, which would have been expected in a case of extensive mitral disease, and the contrac. tion of the pulmonary artery might have caused the bruit, or this might have originated in the tricuspid orifice, which was greatly dilated.

Dr. Morris Toxae exhibited a specimen of DISEASE OF THE SPIYAL CORD,

which was taken from a man admitted into hospital for rheumatism. He was soon afterwards attacked with hemiplegia, loss of speech persisting afterwards, and soon followed by epileptiform convulsions. The brain was found perfectly healthy, with the exception of slight thickening of the arachnoid, but several portions of the spinal cord were found of very firm consistence, very translucent, and quite deficient in natural nerve tissue. A great number of corpora amylacea were found in these parts. The case was thought to be one of epileptic hemiplegia, the epilepsy being due to irritation propagated upwards from the diseased spinal cord.

Mr. Mason showed a case of

SCIRRHOUS CANCER OF THE PROSTITE GLAND.

The patient had suffered from pain in passing urine for three years, the symptoms having commenced a few weeks after an accident. About a year before his death he began to pass blood. A sound detected no stone. There were enlarged inguinal and lumbar glands. He died worn out by suffering. On post-mortem examination the prostate was found replaced by a hard mass, which was thought to be an example of scirrhous cancer. Mr. Mason had examined the prostate microscopically, and had had the assistance of Dr. Johnson. Both agreed that it was scirrhus.

Mr. Thompsor remarked on the great rarity of scirrhus in this organ.

The specimen was referred to Dr. Murchison, Mr. Thompson, and Mr. Mason for report.

Dr. Axprew exhibited a specimen of MALIGNANT DISEASE OF THE UTERUS,

which had in great part sloughed. The character of the tumour had been doubtful during life, but was proved by the occurrence of similar disease in the lungs.

Dr. GREENHALGH read the notes of the case during life, which had been sent into hospital as being a case of ovarian disease. The tumour was of very large size, and occasionally presented fluctuation, from the effusion of blood into the uterus and the tumour. The cancer in this case involved only the fundus and body of the uterus, a peculiarity which Dr. West says that he has only seen three times, and Dr. Greenhalgh only the same number.

Mr. BrookE showed a specimen of

$$
\text { PEDUACCLATED FATTY TUMOUR, }
$$

which he had removed from the thigh of a mictdle-aged woman. The tumour had been punctured by the patient eleven year: ago, under the idea that it was an abscess. This part had grown out of proportion to the other, and Mr. Brooke believed that it had here undergone cancerous degeneration.

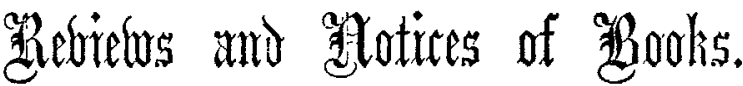

Gunshot Wounds and other Injuries of Nerves. By S. WeIR Mrtchell, M.D., Geor(rE R. Moreholse, M.D., and WILliarr W. KEEs, M.D., Acting Assistant-Surgeons U.S.A., in charge of U.S.A. Wards for Diseases of the Nerrous System, Turner's-lane Hospital, Philadelphia. Philadelphia: J. B. Lippincott and Co. 1864.

Reflex Paralysis: being Circular No. 6, Surgeon-General's office, Washington. 1864 .

IT is gratifying to know that at least some portion of the misery entailed upon man by the American war may be, and has been, utilized. Surgeons and physicians have learned much from all previous campaigns; but the arrangements of the C.S. Army are such that now the physiologist may also profit, and that more readily than he has been wont to do from the fightings of other nations. By the organization of the U.S. Army Hospital for affections of the nervous system, so complete was this field of study renlered, "that it was not uncommon to find at one time four or five cases of gunshot injuries of any single large nerve "“ and thus phenomena which were once deemed rare and curions grew commonplace as experience was enlarged. There is certainly an advantage in this; but the value of many of the facts recorded in both the works mentioned at the head of this article is diminished by their entire freedom from all pathological anatomy. For such absence, however, no blame can be attached to their anthors; but great credit is dne to the physicians, as not one single patient died! The materials for the study of nerre-injuries consist, therefore, of the careful clinical records of 120 cases during life; with regard to which their authors say, and with justice, that " no labour has been spared in making them as perfect and full as possible."

Chapter II. is devoted to a consideration of the Primary Effects of Wounds or other Injuries of Large Nerves; and it is one the value of which is reduced by the fact that it was "impossible to determine accurately the amount of nerve. lesion in most individual cases." With regarl to the pheno. mena of "shock," we are informed that in every case of wound of nerve-trunks in the legs the injured man fell instantly, but not one lost consciousness; whereas of those who were wounded elsewhere, - thirty-one in number, - only seren "fell instantly," but then they "fell unconscious," and all of these were wouncled in the neck, face, or arms. The phenomena of shock were most marked in injuries to the upper third of the body.

In the third chapter, on Wounds of Nerve-Centres, there is nothing new that is of material importance, unless we except the following lines as a specimen of rather curious phraseology and anatomy:- "Two fragments of bone escaped from the wound: one was a part of the carotid foramen of the third vertebra"!

The most interesting fact in the chapter deroted to Wounds of Spinal Nerves is that recorded in the case of a man whose neck was entered by a ball behind the ramus of the jaw on the right side, the ball escaping in front of the angle of the jaw on the left side; and in whom a month later it was roticed that "the pupil of the right eye was very small, and that of the left eye unusually large," with ptosis of the right eyelid, the ball of which looked smaller than the left. It was also noticed that after walking in the heat the face "became distinctly flushed on the right side only, and pale on the left." These changes the authors refer to "commotion of the spine in the cilio-spinal track."

In the fifth and sixth chapters, on Wounds and other Injuries of Nerve-Trunks, evidence is given to show that contusions may injure nerves so as to cause atrophy, which may involve only certain fibres of a muscle and respect the rest; such atrophy being sometimes accompanied by a contracted state of the muscles. It is also shown that disease may extend along the course of nerve-trunks so as to produce the results of injured nerves in parts remote from those on which the original injury was inflicted.

The effects of wounds on the nutrition of the skin and its appendages are described with care, and are of interest. Early in the case of entire division of the nerves of a part, with total palsy of a whole limb, there is, in most instances, cedema, with thickening and dryness of the skin, and a change in its tint to yellow or pale-brown; the epithelium hangs in patches here and there, and the nails are curved as in tubercular disease. In other cases may be observed that "glossy" skin which was tirst described by VIr. Paget a year ago, and which our authors state does not belong to cases of complete division 